\section{¿Políticas de salud y salud politizada? Un análisis de las políticas de salud sexual y reproductiva en Perú desde la perspectiva de la ética médica, calidad de atención y derechos humanos}

\author{
Health policies and politicized health? An analysis \\ of sexual and reproductive health policies in Peru \\ from the perspective of medical ethics, quality of \\ care, and human rights
}

J. Jaime Miranda 1,2,3

Alicia Ely Yamin 1,4,5

\footnotetext{
${ }^{1}$ Asociación Civil para la Educación en Derechos

Humanos con Aplicación en Salud, Lima, Perú.

2 Department of

Epidemiology and Population Health, London School of Hygiene and Tropical Medicine, London, U.K.

${ }^{3}$ Facultad de Salud Pública y Administración, Universidad Peruana Cayetano Heredia, Lima, Perú.

${ }^{4}$ Petrie-Flom Fellow on Global Health and Human Rights, Harvard Law School, Boston, U.S.A.

${ }^{5}$ Department of Health Policy and Management Harvard School of Public Health, Boston, U.S.A.

Correspondencia J. J. Miranda

Asociación Civil para la

Educación en Derechos

Humanos con Aplicación en

Salud.

Moquegua 145, Santa

Patricia 2, La Molina

Lima 12, Perú.

jaime.miranda@edhucasalud. org

jaime.miranda@lshtm.ac.uk
}

\section{Introducción}

Health professionals view medical ethics as a discipline that provides the basis for more adequate patient care. In recent years the concepts of quality of care and human rights - with their attending discourses - have joined the concept of medical ethics among the paradigms to consider in care for humans both at the individual and health policy levels. The current study seeks to analyze such paradigms, based on a case study of sexual and reproductive health policies in Peru in the last 10 years.

Sexual and Reproductive Health; Health Policy; Quality of Health Care; Medical Ethics; Human Rights
Las acciones que se plantean para proteger y promover un buen estado de salud, así como los actores necesarios para llevar tales tareas a cabo, dependen de la manera en que se conceptualiza el término "salud". Este ensayo presenta una breve descripción de las políticas de salud vinculadas a la salud sexual y reproductiva que tuvieron lugar en Perú en los últimos 10 años, continuando con un análisis de las implicancias y repercusiones que distintos paradigmas - o puntos de partida para comprender y conceptualizar la salud - nos ofrecen. Los conceptos de ética médica, calidad de atención y derechos humanos han sido escogidos para tal análisis, dada su relevancia e importancia en el cuidado de la salud de nuestros congéneres. Concretamente, estos paradigmas nos ofrecen maneras distintas de formular, ejecutar y evaluar las políticas de salud, además de comprender las relaciones entre los diferentes actores, a distintos niveles, involucrados en el proceso. Utilizando como base trabajos previos sobre las políticas de salud sexual y reproductiva en el Perú 1,2 y críticas a los modelos clásicos de la prestación de servicios de salud, este documento enfatiza la importancia de crear y promover una cultura de salud como derecho, salud como derecho humano. 


\section{Salud sexual y reproductiva en contexto}

Los indicadores de mortalidad materna y mortalidad infantil son utilizados frecuentemente en el área de salud sexual y reproductiva. De un total de 27 millones de peruanos, las mujeres en edad reproductiva constituyen un $25 \%$ de la población total 3 . La tasa de mortalidad materna en Perú para el período previo al año 2000 fue de 410 muertes por 100.000 nacidos vivos. Perú y Bolivia, cuya tasa es de 420 muertes, son los países con peores indicadores de mortalidad materna en América del Sur 4

Un niño que nace en Perú, donde la mortalidad infantil es de 32 por 1.000 nacidos vivos, tiene más del doble de posibilidades de fallecer antes de llegar a su primer año de vida que un niño nacido en Chile y más de 10 veces cuando se compara con las probabilidades de sobrevivir en Suecia o Japón 4 .

Las cifras promedio a nivel nacional enmascaran una realidad de indicadores divergentes y de disparidades en donde las zonas de los Andes y de la Amazonía figuran entre las más desfavorecidas, además de una clara y marcada diferencia urbano-rural 5 .

Un aspecto importante en el campo de la salud sexual y reproductiva lo constituye, por su vinculación como una de las causas de mortalidad materna, la problemática del aborto y de los embarazos no deseados. A pesar de que éstos no son parte de la discusión en el presente trabajo, vale la pena mencionar algunos hallazgos que permitan poner en contexto la complejidad de la salud sexual y reproductiva en Perú.

El aborto inseguro representa un 16\% del total de muertes maternas. Existen aproximadamente 66 abortos por cada 100 nacidos vivos en el país, en donde el aborto es ilegal. Como mínimo, 30\% del total de abortos resulta en complicaciones 6 . Adicionalmente, se estima que $60 \%$ del total de embarazos son no deseados, y un $25 \%$ de mujeres sexualmente activas en edad reproductiva en el Perú no están adecuadamente protegidas contra un embarazo no deseado ${ }^{6}$. Esta situación añade variables distintas al no menos complejo problema de la salud sexual y reproductiva en Perú.

\section{5-1998: el control poblacional como meta}

No es nuevo el sostener que en las últimas décadas muchos países en desarrollo han tenido, y siguen teniendo, presión internacional con el fin de aplicar modelos económicos neoliberales a través de los llamados "programas de ajustes estructurales" (del Inglés, structural adjustment programs). Tales modelos formulan que los gobiernos deberían permitir que el mercado se "haga cargo" de la provisión de servicios básicos a los ciudadanos, incluyendo salud. La oferta y la demanda brindarían las condiciones necesarias para que los servicios necesarios sean otorgados al consumidor de acuerdo con sus demandas y preferencias. El mercado brindaría el mecanismo más eficiente para la asignación de los recursos a los servicios sociales básicos. Al mismo tiempo, la privatización de tales servicios permitiría que se tenga un Estado con menos gastos, y en el futuro le permitiría ser más eficiente y estar en una mejor posición para afrontar sus compromisos fiscales, sobretodo, la deuda externa.

El resultado de tal modelo, aplicado con diferente rigurosidad en diferentes países por el Fondo Monetario Internacional, conjuntamente con bancos multilaterales para el desarrollo, ha sido en la práctica no menos que catastrófico ${ }^{7}$. En muchos países, incluyendo Perú, el sector salud ha sido uno de los primeros en ser afectados al no recibir financiamiento adecuado por parte del gobierno ${ }^{8}$.

El beneficio potencial de tales modelos y predicciones no ha llegado, y lo que es peor, la salud de millones de personas - sobretodo aquellas fuera de la "economía productiva" - se ha visto afectada al encontrarse atrapados en un círculo vicioso de pobreza-enfermedad que conduce a mayor pobreza-enfermedad.

Sin ir muy lejos, en Perú entre los años 1997 y 2001, el $80 \%$ del total de gastos privados en salud - aquellos que no son gastos por parte del Gobierno - provienen directamente de los bolsillos de las personas ${ }^{4}$. En un país con más de la mitad de la población viviendo en niveles de pobreza $-57,4 \%$ en 1991 y $54,1 \%$ en el año $2000{ }^{9}$ - no es difícil predecir que los niveles de salud de tales sectores de la población se verán afectados de manera importante, además de otras esferas, como educación, por sacrificios, usualmente invisibles, llevados a cabo para poder sobrevivir. Concretamente, si no puedes pagar para tener un nivel adecuado de salud, dejas de estudiar, no puedes trabajar y las oportunidades de progresar en la vida se ven severamente afectadas.

Este modelo de salud tiene también consecuencias distintas a nivel de género. Son las mujeres las que cuidan el hogar y la familia, son las mujeres las que asumen mayor responsabilidad cuando el Estado abandona su rol de proveer servicios básicos, y son ellas mismas las que tendrán niños, aquellos que eventualmente formarán parte del sector laboral o parte del sector desempleado 10,11 . 
En Perú, en la década de los 90, durante el mandato de Albero Fujimori (1990-2001) se llevaron a cabo las políticas del modelo de desarrollo neoliberal, las mismas que incluyeron la “modernización” del sector salud. En este mismo período se consideró que el crecimiento poblacional era uno de los factores limitantes para el desarrollo económico del país, vale decir, los millones de pobres de Perú traían al mundo más individuos pobres.

Interesante como suena, en un país en donde los temas de salud no son parte de la agenda de los gobernantes, ni obtienen titulares de primera plana, el mismo Fujimori en julio de 1995 anunció que la planificación familiar sería una prioridad para el gobierno. Poco después, el Congreso de la República aprobaría y legalizaría la esterilización quirúrgica como un método de planificación familiar.

La meta era reducir la tasa de crecimiento poblacional anual a una cifra alrededor de 1,17 para el período 1995-2000 12. Dicho indicador mostraba un descenso progresivo que se había iniciado en las décadas anteriores, siendo 2,8 en el período 1961-1972, 2,0 entre 1981-1993, alcanzando 1,7 entre 1993-2002, incluyendo este último el período de gobierno de Fujimori. En otras palabras, el esperar la tendencia de reducción del crecimiento poblacional de acuerdo a las tendencias observadas no sería suficiente. Entonces, la meta sería por el contrario acelerar el proceso. El mismo Fujimori tomó un interés personal en tal programa y, como cualquier plan que necesita mostrar resultados, algunas metas fueron establecidas para poder monitorear los avances y evaluar los logros de este programa, entre ellas el número de mujeres esterilizadas. Ha sido indicado que el proceso de monitoreo era implementado desde el mismo Palacio de Gobierno 13 .

Producto de dicho programa, más de 250.000 "beneficiarias" fueron esterilizadas 14, la mayoría sin un proceso adecuado de consentimiento informado, producto de las presiones tácitas para cumplir con las metas establecidas. Vale la pena resaltar que la mayoría de dichas mujeres correspondían al estrato socioeconómico bajo y eran a la vez procedentes de zonas rurales con alta concentración de poblaciones indígenas pobres.

En 1998 y 1999, la revelación de que esterilizaciones quirúrgicas fueron llevadas a cabo de manera involuntaria como parte de dicho programa produjo un gran escándalo, no sólo a nivel local, sino también a nivel internacional. En particular, un grupo de representantes del congreso de los EEUU establecieron su propia comisión investigadora, debido a que la Agencia para el Desarrollo Internacional de dicho país (USAID
- US Agency for Internacional Development) había financiado directamente, y en gran parte, tal programa de salud.

\section{1-2003: introduciendo agendas conservadoras como parte del remedio}

Desde que el Presidente Alejandro Toledo asumió el poder en 2001, el Ministerio de Salud fue dominado por elementos conservadores religiosos, quienes reformularon las políticas de los programas relacionados con la salud sexual y reproductiva de acuerdo con una agenda ideológica específica. Tal mandato se reflejó principalmente, aunque no únicamente, en un bloqueo al acceso a métodos anticonceptivos e imponiendo penalidades más severas para los casos de aborto 1,2,15,16. En parte, dichos cambios en la posición del gobierno en relación a la salud sexual y reproductiva eran justificados como respuesta a la mala experiencia durante la época de las esterilizaciones.

Casi inmediatamente después de la asunción al mando del Presidente Toledo se formaron dos comisiones para investigar los casos de las esterilizaciones - uno dentro del propio Ministerio de Salud y uno dentro del Congreso de la República, el mismo que fue promovido por políticos vinculados a organismos y agendas religiosas conservadoras. Ambas comisiones encontraron hallazgos similares. La comisión del Congreso llegó incluso a calificar las esterilizaciones llevadas a cabo como parte de una política de "genocidio". Esta misma comisión cita, por su parte, figuras de procedimientos de esterilización quirúrgica realizados de manera involuntaria o forzada del orden de 90 veces más altas que aquellas reportadas por grupos feministas y la misma Defensoría del Pueblo 17.

Del mismo modo que el deseo de controlar el crecimiento poblacional para facilitar el desarrollo económico era un reflejo del momentum de tendencias transnacionales, también lo fue el fortalecimiento de influencias religiosas conservadoras en sector salud del Perú. Por ejemplo, al mismo tiempo y progresivamente, el gobierno de los EEUU inició el bloqueo de financiamiento de programas que promovían la salud sexual y reproductiva de manera contradictoria a sus principios ideológicos. Esta creciente influencia religiosa conservadora de parte del gobierno de los EEUU conllevó, en la práctica y entre otras cosas, a reducir el financiamiento de organismos técnicos de la Naciones Unidas, tales como el Fondo de Población de las Naciones Unidas 18,19.

Específicamente, las políticas restrictivas del gobierno de los EEUU, en cuanto a financiamien- 
to a instituciones extranjeras en temas de salud sexual y reproductiva, incluyen no solamente limitaciones en la provisión de tales servicios, sino también en la provisión de información en temas relacionados al aborto, política comúnmente conocida como la Ley Global de la Mordaza, o "Global Gag Rule" en Inglés. Asimismo, toda información relacionada con estrategias de prevención en VIH/SIDA deberá poner énfasis en la abstinencia, incluso a costa de iniciativas de educación sexual y provisión de condones. Estos hechos han promovido marcadas críticas, por parte de líderes y expertos en los sectores políticos y de salud, a nivel internacional 20. Resulta claro, entonces, que todo organismo que pretenda acceder a los recursos de la cooperación externa de los EEUU deberá someterse a sus políticas y agendas específicas en temas de reproducción en los países en vías de desarrollo, vale decir, anti-aborto y pro-abstinencia.

\section{Paradigmas para comprender la prestación de servicios de salud: puntos de partida diferentes nos dan interpretaciones diferentes}

\section{Bioética y ética médica}

La profesión médica tiene una larga historia en la promoción de una conducta ética hacia los pacientes. El Juramento Hipocrático, promulgado por el antiguo filósofo griego y médico Hipócrates, se constituye como el código que guía la conducta ética y responsable de los médicos para con los pacientes. En los últimos años, reconocidos cuerpos médicos locales e internacionales siguen trabajado en base a declaraciones y códigos de ética, los mismos que tienen que ver principalmente con las responsabilidades de parte de la profesión médica en el cuidado de los pacientes 21,22 .

El paradigma tradicional de ética médica asume una relación "dual" o "diádica" entre médico y paciente - refiriéndose a una comunicación bidireccional que no admite influencias externas - que usualmente se encuentra largamente divorciada del contexto social.

La mayoría de discusiones éticas en medicina examinan cómo decisiones "puramente" clínicas podrían afectar la vida de los pacientes. El conocimiento especializado del médico y la vulnerabilidad de los pacientes demandan una relación fiduciaria, basada en la confianza. De ella se desprenden, como es de esperarse, una serie de obligaciones éticas básicas: beneficencia, no maleficencia, autonomía y justicia 23. Bajo este modelo, el cumplimiento de tales obligaciones es regulado y evaluado de manera interna - o sea, entre los mismos profesionales - por los respectivos organismos o agrupaciones médicas.

La "voz" de los pacientes - aquellos que proceden de culturas diferentes y específicas, además de mantener concepciones particulares sobre su propio bienestar - es raramente escuchada en discusiones éticas profesionales. Vale la pena notar que aquellos factores que no forman parte de la interacción médico-paciente - incluyendo las influencias que tienen la cultura y los sistemas tradicionales de salud en como se definen y promueven el bienestar en diferentes individuos y sociedades - no son contemplados cuando se trata el tema de ética médica.

Es por ello que, los diferentes modelos de relación médico-paciente desarrollados por especialistas en ética de países desarrollados parecerían tener una relevancia limitada en las áreas rurales del Perú. Prejuicios culturales e históricos conllevarían a que el doctor cumpla no sólo un rol paternalista, sino también "civilizador". La medicina occidental, así como la lógica inherente a dicha práctica, parecería que tuviese un efecto civilizador sobre las costumbres de los pacientes indígenas o campesinos. Además de ello, muchos pacientes no toman individualmente decisiones sobre su salud, así como tampoco tienen la opción de elegir donde recibir la atención debida para el cuidado de su salud. Dado que en teoría el poder de los médicos es regulado por medio de estándares éticos, aquellas relaciones de poder que no son consideradas parte de la relación médico-paciente - a pesar que por si mismas afectan el bienestar del paciente - no están sujetas a escrutinio en este modelo.

En relación a las esterilizaciones que se llevaron a cabo sin un debido consentimiento, un modelo basado en ética médica en un vacío buscaría la sanción del médico practicante, dejando de lado una evaluación de la política per se o de como ésta tendría un efecto sobre la conducta a nivel individual del profesional de salud. Continuando en el marco ético, el primer Ministro de Salud durante el gobierno de Alejandro Toledo, Dr. Luis Solari de la Fuente, tomó acciones que brindaban una clara apreciación de las esterilizaciones como un tema de "lealtad dual". Esto es, el deber de los profesionales de salud para con los pacientes se encontraba en una situación de conflicto con las políticas perversas de la administración previa 24. Solari propuso una Ley de Objeción de Conciencia, la misma que hubiera permitido a los médicos rehusar brindar atención de salud en situaciones moralmente conflictivas, sobre la base de objeciones religiosas $\mathrm{u}$ otras creencias. Solari-de la Fuente 25 sostenía que la causa de las esterilizaciones involuntarias se encontraba en el 
hecho de que "el profesional de salud no tenía la libertad para hacer lo que su conciencia y entrenamiento le decian".

La solución propuesta buscaba, aparentemente, empoderar a los médicos con el fin último de asegurar y salvaguardar una mejor atención a los pacientes. Sin embargo, el brindar a los médicos toda la aprobación para rehusar tratar a un individuo hubiera potencialmente puesto en una situación compleja - y quizás desfavorecedora - el cuidado de los pacientes, como por ejemplo en casos de emergencia en donde un único médico puede ser la única fuente de atención disponible en muchas áreas rurales del Perú. Aquellos que han explorado el problema de "lealtad dual", aquel que surge de un conflicto de intereses entre la lealtad hacia el paciente y hacia el Estado, enfatizan la necesidad de expandir las concepciones éticas tradicionales más allá de la relación interpersonal entre médico y paciente, debiendo considerarse el contexto en el cual se presta la atención 26,27.

\section{Calidad de atención}

Cambios en la salud pública han conducido a una mayor atención al tema de calidad de atención. La calidad de atención, incluyendo el tratamiento de pacientes por parte de sus proveedores, ha sido un componente crítico en el mejoramiento de la provisión de servicios de salud en Perú y alrededor del mundo. El "paradigma de calidad de atención" al que nos referimos en este trabajo emerge del campo de la salud pública, teniendo marcados contrastes con la medicina clínica.

Las nociones económicas de eficiencia, costo-efectividad y mercado han jugado un rol sustancial, aunque no exclusivo, en el desarrollo de modelos para la evaluación del concepto de calidad de atención. Los análisis de costo-efectividad son una de las herramientas, entre otras, sobre las que se basan las decisiones acerca de la provisión de servicios de calidad. Vale la pena notar que tales análisis varían dependiendo del punto de vista desde el cual se realice el análisis, sea en términos de costo para los pacientes o usuarios, para los servicios de salud o para la sociedad. Los resultados serán diferentes, dependiendo de las presunciones en las que se basen los cálculos, dado que la percepción de los costos y las prioridades son diferentes entre los distintos tipos de actores, notándose además diferencias intra e inter contextos.

Este constructo ubica al acto médico en el contexto del sistema de salud, donde los actores pasan a jugar el rol de "proveedores" y "clientes", refiriéndose a profesionales de salud y pacientes, respectivamente. En un nivel de sistemas, la atención del "cliente" no debería dejarse al deseo o discreción del proveedor individual, por el contrario debería cumplir con ciertos requisitos y regirse por ciertas normas. Algunos de estos son de tipo técnico, con el fin de mejorar la capacidad de los servicios, mientras que otros están más directamente relacionados con la manera en que los pacientes son tratados y la gestión del establecimiento.

En Perú, en respuesta al criticismo por el respaldo al Programa de Planificación Familiar, USAID encargó un estudio sobre el tema de las esterilizaciones involuntarias a Population Council, quienes analizaron el problema desde una perspectiva de calidad de atención. El reporte preparado por Population Council limitó la discusión de los abusos cometidos, así como la inapropiada intervención del Estado, a una esfera totalmente personal en la relación entre médicos y pacientes. El reporte concluyó que, inter alia, habían existido algunas brechas en algunas instancias de confidencialidad, comunicación e información entre proveedores y clientes, así como a distintos niveles del sistema, lo que condujo a un estándar pobre en la calidad de atención, incluyendo la muerte de un número de mujeres. Concretamente, hubo error en el cálculo de los costos y beneficios de proveer incentivos y sanciones a los profesionales de salud que llevarían a cabo las esterilizaciones, presentándose consecuencias secundarias. El reporte incluía también algunas recomendaciones acerca de cómo el Ministerio de Salud podría rectificar estos errores en el futuro 28 .

\section{Derechos humanos}

El marco de los derechos humanos sitúa la salud, y por ende la práctica de la medicina, como parte de relaciones sociales e instituciones que progresan, o afectan, la justicia social. En esto el personal de salud tiene un rol en la provisión de atención accesible, aceptable y de calidad a todos los pacientes sin discriminación alguna 29.

La atención ética de los pacientes, así como la certeza de la provisión de servicios con calidad, constituyen partes integrales del derecho a la salud. A pesar de ello, el paradigma de los derechos humanos, por el contrario, va más allá. El marco de derechos apuesta por empoderar a los pacientes para que no se vean a sí mismos tan sólo como pacientes con un diagnóstico determinado, ni tampoco como los consumidores de los servicios de salud que deberían recibir calidad, sino como ciudadanos poseedores de derechos capaces de hacer demandas que van más allá de los paquetes de servicios. La salud bajo este paradigma es entendida como una precondición y 
una reflexión de un ejercicio activo de la ciudadanía. Resulta lógico, por ello, que la perspectiva de derechos humanos preste atención a los grupos socialmente marginados que se ven excluidos tanto de la salud como de la posibilidad de participar como ciudadanos y ciudadanas plenos en su sociedad 30 .

En contraste con las posturas tomadas por el entonces Ministro Solari o Population Council, el grupo de mujeres del Comité Latinoamericano para la Defensa de los Derechos de la Mujer (CLADEM) preparó un reporte en donde el problema se conceptualizó desde una perspectiva de derechos humanos 31. El CLADEM conceptualizó los eventos no solamente en el contexto de sistemas de salud, sino en la sociedad amplia, e insistió en una política más amplia y en cambios legislativos, así como en reparaciones para las víctimas y sus familias. Una aproximación de derechos humanos entiende el problema fundamental de las esterilizaciones forzadas como minar la capacidad de elección y la dignidad de mujeres que eran en su gran mayoría indígenas.

Es así que, en un marco de derechos humanos, la protección del derecho a la salud debe estar vinculada a acciones que involucren otros sectores, además del sector salud. Esto incluye la provisión de educación, acceso a un sistema judicial, libertades civiles, derechos laborales, y la oportunidad para participar políticamente. Tales requisitos coinciden con la nueva ola de pensamiento, proveniente del campo de la salud pública, en relación con las cualidades necesarias para determinar qué intervenciones serían las más efectivas.

Aparte de constituir por si mismo un paradigma conceptual diferente en el cual la salud se entiende como parte de amplias y diversas condiciones sociales de la vida de la gente, los derechos humanos también conforman un cuerpo de leyes. Como tales, entonces, imponen ciertas obligaciones a los Estados en relación con la salud, además de una serie de otros aspectos relacionados con el bienestar social. Los gobiernos que ratifican los tratados internacionales son legalmente responsables ante la sociedad acerca del cumplimiento de ciertas obligaciones para sus ciudadanos.

Tales responsabilidades implican, en primer lugar, que la determinación de ciertas políticas y la implementación de programas respecto al sistema de salud nacional deberán ser guiadas por ciertos principios fundamentales, en vez de ser simplemente un asunto de negociación política. En segundo lugar, deben ponerse al alcance los recursos necesarios para aquellos agraviados por la falta - o falla - del Estado en honorar tales principios. El discurso de los derechos, además de la vinculada responsabilidad legal del gobierno en respetar, proteger y satisfacer sus obligaciones con respecto a la salud impulsa un diálogo abierto y mantiene una atención pública, que de otra manera podría pasar desapercibida 32 .

De allí que, el considerar a los derechos humanos simplemente como un grupo más de principios de ética o guías para una atención con calidad, sería una completo error entender la concepción de la dimensión de los derechos 33,34.

Easley et al. 34 (pp. 1922-3) presentan la siguiente diferenciación: "Los derechos humanos tienen una base moral y filosófica, con un esbozo en lo que significa e implica ser un ser humano, y en procesos legales y políticos por los cuales las sociedades humanas son gobernadas. La ética, como una de las ramas de la filosofía que detalla primariamente con la definición de acciones moralmente correctas, opera en un área del discurso relacionada, pero a la vez diferente. Como profesionales de salud pública, encontramos la ética como descripciones de o prescripciones para comportamientos morales hacia nuestros pacientes o clientes. Estos mandatos éticos son codificados frecuentemente por organizaciones profesionales y sirven como guías para la toma de decisiones en situaciones prácticas específicas. Los derechos humanos también son resultado del juicio moral acerca del buen comportamiento, pero en la forma de normas bajo mutuo acuerdo, articulando los reclamos de grupos e individuos y la obligación relacionada del Estado".

En Perú, por ejemplo, CLADEM buscó que se compensen a familias de las víctimas, así como el establecimiento de precedentes legales de responsabilidad institucional. Junto con otros grupos de derechos humanos CLADEM peleó por un caso emblemático, en el que una mujer fue forzada a un procedimiento de esterilización, falleciendo poco después. Después de que el caso no fue favorable en el sistema legal peruano, estos grupos condujeron de manera satisfactoria una petición a la Comisión Interamericana de Derechos Humanos 35.

\section{¿Después del análisis, qué? Educación en derechos humanos y salud para los profesionales de salud}

Retomando una de las afirmaciones hechas al inicio del presente documento, es importante tener claro que las acciones que se plantean para proteger y promover un buen estado de salud, así como los actores necesarios para llevar tales tareas a cabo, dependen de la manera en que se conceptualiza el término "salud". La conceptualización de la salud - la manera como se entiende y 
define salud - es un proceso. En tal proceso intervienen diferentes factores, tanto a nivel personal como grupal o social, además de las condiciones del entorno y el estatus social. Todos ellos "darán forma" a lo que se entiende por salud y estado saludable. Específicamente, variables importantes para la concepción de salud son educación y estrato socioeconómico.

En relación al presente trabajo en donde se discuten paradigmas diferentes, es de esperar que sean diferentes los actores participantes según el paradigma estudiado. Entre ellos se encontrarán bioéticos, médicos generales y especialistas, salubristas o profesionales de salud pública, administradores de salud, abogados y empleados del gobierno. La concepción de salud, o mejor dicho, la manera en que se entiende salud difiere entre ciudadanos comunes, entre profesionales con distinto entrenamiento, $\mathrm{y}$ más aún entre aquellos directamente vinculados con el sector salud como aquellos mencionados anteriormente. Ha sido demostrada que la disquisición clásica, y completamente errónea, de que los derechos humanos son temas sólo para abogados y la salud sólo para los médicos ya no es aplicable cuando hablemos de salud en el siglo XXI. Ambas son disciplinas que requieren las aportaciones de ambas profesiones.

Como se ha visto anteriormente en el análisis de las políticas de salud sexual y reproductiva que se dieron en Perú, los enfoques de ética médica y calidad de atención presentan dificultades en distintos niveles del proceso de evaluación de políticas de salud pública. El marco de derechos humanos, por su parte, es un marco complementario y alternativo que permite identificar - y, de aplicarse apropiadamente, podría también prevenir - algunas de las dificultades observadas con los otros enfoques discutidos. Uno de los principios fundamentales de la práctica de la medicina, establecidos en el Juramento Hipocrático, es primun non nocere, primero no hacer daño. Muchos de los involucrados en el diseño y ejecución de políticas públicas de salud concordarían en que mucho mejor sería prevenir aquellas situaciones que, potencialmente, resulten en violaciones de derechos humanos producto de la puesta en práctica de políticas o programas, incluyendo aquellas políticas relacionadas al sector salud.

Un mejor uso de los paradigmas expuestos sería el prevenir situaciones conflictivas para los proveedores de salud antes que utilizarlos con el propósito de "identificar" y "sancionar" a responsables. Por ende, parte primordial de tal proceso debería incluir la participación de los profesionales de salud en general en debates abiertos sobre el ámbito y repercusión de los campos de ética, calidad de atención y, sobretodo, derechos humanos en la salud de las personas.

El punto crítico para el progreso de la conceptualización de la salud hacia una salud que respete los derechos de todas las personas requiere la educación de médicos, enfermeras, salubristas y todo profesional de salud en temas de derechos humanos. Lamentablemente, este progreso se ve limitado por la inacción y falta de atención, en casi todos los ámbitos, de aquellos involucrados con la salud y los derechos humanos: el sector público, la academia y las organizaciones de la sociedad civil.

El sector público es responsable, pues no considera la salud como un derecho. La academia es responsable, porque no inculca a sus estudiantes el entender la salud como un derecho. La sociedad civil es responsable, porque no exige que el profesional de salud entienda, como ellos lo entienden, la salud como un derecho. Mientras existan estos distanciamientos, el progreso hacia la realización de la salud como derecho está lejos de ser alcanzado 18,19,36,37,38. Una exposición temprana a discusiones abiertas y comparaciones de diferentes paradigmas, resultaría beneficiosa para las nuevas generaciones de médicos y demás profesionales de salud, para los pacientes y ciudadanos, y, sobretodo, para la sociedad en general.

\section{Conclusión}

Las políticas de salud sexual y reproductiva del Perú han sido analizadas desde diferentes ángulos - el de ética, calidad de atención y derechos humanos - resaltando en cada uno de ellos los vacíos que ocurren, y mostrando áreas de convergencia y sinergia, especialmente en el campo de los derechos humanos.

En Perú, un país con una historia de gobiernos represivos podría existir resistencia a tocar el tema entre los diseñadores de programas, precisamente porque hablar de derechos humanos significa retomar al punto de las obligaciones y responsabilidades del Estado. Por ello, muchos activistas en temas de salud prefieren discutir sobre tópicos como "calidad de atención" y "ética médica" o "valores". Es por ello que nos parece importante resaltar por qué estos modelos no pueden simplemente ser sustituidos por una disertación de derechos humanos.

Las mejoras en la calidad de atención o la educación en ética médica per se hacen poco para revertir las estructuras de poder social que niegan la voz de las personas con respecto a su propia salud. El marco de los derechos humanos reta a las atrincheradas estructuras de poder que 
permiten la existencia y permanencia de grandes inequidades en los estándares de salud en las poblaciones, tanto internamente en un mismo país, como entre diferentes países. En este contexto de adversidades, este marco liga el derecho a la sa- lud y el rol del profesional de salud en una lucha mayor en pro de la democracia y la justicia social, de allí la importancia de este trabajo exposición y de la educación en derechos humanos a los futuros profesionales de salud.

\section{Resumen}

La ética médica es considerada entre los profesionales de salud como la disciplina que proporciona las bases para brindar una atención adecuada a los pacientes. En los últimos años, los conceptos de calidad de atención y derechos humanos - así como sus diversos discursos acompañantes - se han sumado al concepto de ética médica entre los paradigmas a tener en cuenta en la atención de las personas, tanto a nivel individual, así como a nivel de políticas de salud. El presente trabajo busca analizar tales paradigmas, utilizando como estudio de caso las políticas de salud sexual y reproductiva que se dieron en Perú en los últimos 10 años.

Salud Sexual y Reproductiva; Política de Salud; Calidad de la Atención de Salud; Ética Médica; Derechos Humanos

\section{Colaboradores}

J. J. Miranda y A. E. Yamin concibieron el artículo. J. J. Miranda escribió la primera versión del artículo, A. E. Yamin revisó el artículo y contribuyó con contenidos intelectuales importantes. Ambos autores revisaron la versión final del mismo y dieron su aprobación para publicación.

\section{Referencias}

1. Coe AB. From anti-natalist to ultra-conservative: restricting reproductive choice in Peru. Reprod Health Matters 2004; 12:56-69.

2. Miranda JJ, Yamin AE. Reproductive health without rights in Peru. Lancet 2004; 363:68-9.

3. Instituto Nacional de Estadística e Informática.Perú en cifras. http://www.inei.gob.pe/web/perucifras home.asp (accedido el 11/Ago/2007).

4. World Health Organization. The World Health Report 2004 - changing history. Geneva: World Health Organization; 2004.

5. Miranda JJ, Malca R, Bedriñana E, Loayza E. Culturally-based health promotion programmes. Lancet 2002; 359:262-3.

6. Fernando D. El aborto clandestino en el Perú. Hechos y cifras. Lima: Centro de la Mujer Peruana Flora Tristán/Pathfinder International; 2002.

7. Stiglitz J. Globalization and its discontents. New York: WW Norton; 2002.

8. Ooms G, Schrecker T. Expenditure ceilings, multilateral financial institutions, and the health of poor populations. Lancet 2005; 365:1821-3.

9. Instituto Cuanto. Encuesta Nacional sobre Medición de Niveles de Vida (ENNIV) 2000. In: Webb R, Fernandez-Baca G, editores. Anuario estadístico: Perú en números 2003. Lima: Instituto Cuanto; 2003.

10. Evers B, Juárez M. Understanding the links: globalization, health sector reform, gender and reproductive health. New York: Ford Foundation; 2003. 
11. Ewig C. Gender equity and neoliberal social policy: health sector reform in Peru [Tesis de Doctorado]. Chapel Hill: University of North Carolina at Chapel Hill; 2001.

12. Population: brickbats and bouquets for Peruvian policies. Inter Press Service 1997; 14 nov.

13. Gobierno ordenaba a médicos cumplir cuotas de mujeres esterilizadas. La República 2001; 23 sep.

14. Bosch X. Former Peruvian government censured over sterilisations. BMJ 2002; 325:236.

15. Coe AB, Jacobson J. Government extremists in Peru further undermine reproductive rights. Tahoma Park: Center for Health and Gender Equity; 2002.

16. Defensoría del Pueblo. Informe defensorial no ${ }^{\circ} 69$. La aplicación de la anticoncepción quirúrgica y los derechos reproductivos III. Lima: Defensoría del Pueblo; 2002.

17. Subcomisión Investigadora de Personas e Instituciones Involucradas en las Acciones de Anticoncepción Quirúrgica Voluntaria. Informe final sobre la aplicación de la anticoncepción quirúrgica voluntaria (AQV) en los años 1990-2000. Lima: Congreso de la República del Perú; 2002.

18. Yamin AE. Challenges and possibilities for innovative praxis in health and human rights: reflections from Peru. Health Hum Rights 2002; 6:35-64.

19. Yamin AE. Embodying shadows: tracing the contours of women's rights to health. In: Gordon N, editor. From the margin of globalization: critical perspectives on human rights. New York: Lexington Books; 2004. p. 223-57.

20. Boseley S, Goldenberg S. Brazil spurns US Aids help. Move resists effort by Washington to impose religious right's agenda. Guardian Weekly 2005; May 13-19:32.

21. Colegio Médico del Perú. Código de ética y deontología. http://www.cmp.org.pe/doc_norm/codi go_etica_cmp.pdf (accedido el 11/Ago/2007).

22. World Medical Association. International code of medical ethics. http://www.wma.net/e/policy/ c8.htm (accedido el 11/Ago/2007).

23. Ugarte-Gil CA, Miranda JJ, Echevarría J. Principios éticos y derechos relacionados con el tratamiento antirretroviral en los pacientes con VHI-SIDA. Lima: Instituto de Medicina Tropical Alexander von Humbdolt/Universidad Peruana Cayetano Heredia; 2004.

24. Yamin AE, Miranda JJ. Ética, calidad de atención y derechos humanos. Idéese 2002; 1:35-42.

25. Solari-de la Fuente L. Proyecto de Ley que garantiza a los profesionales de la salud la absoluta libertad profesional para ejercer el derecho de objeción de conciencia. Proyecto $n^{\circ}$. 116/2001-CR. Lima: Congreso de la República del Perú; 2001.
26. International Dual Loyalty Working Group. Dual loyalty \& human rights in health professional practice; proposed guidelines and institutional mechanisms. Boston: International Dual Loyalty Working Group; 2002.

27. Rubenstein LS. Promover los derechos humanos como imperativo de la medicina. In: Memorias del Primer Taller Latinoamericano Permanente "Derechos Humanos y Salud: Encontrando los Lazos". Lima: Asociación Civil para la Educación en Derechos Humanos con Aplicación en Salud; 2001. p. 20-3.

28. León F. Peru: providers' compliance with quality of care norms. Lima: Population Council; 1999.

29. Committee on Economic Social and Cultural Rights. The right to the highest attainable standard of health. Geneva: Committee on Economic Social and Cultural Rights, United Nations; 2000. (General Comment, 14).

30. Miranda JJ, Farmer P. Social exclusion must be considered in global terms. BMJ 2001; 323:1370.

31. Tamayo G. Nada personal: reporte de derechos humanos sobre la aplicación de la anticoncepción quirúrgica en el Perú 1996-1998. Lima: Comité Latinoamericano para la Defensa de los Derechos de la Mujer; 1999.

32. Yamin AE. Protecting and promoting the right to health in Latin America: selected experiences from the field. Health Hum Rights 2000; 5:117-48.

33. Chapman A. A "violations approach" for monitoring the International Covenant on Economic, Social and Cultural Rights. Hum Rights Q 1996; 18:23-66.

34. Easley CE, Marks SP, Morgan Jr. RE. The challenge and place of international human rights in public health. Am J Public Health 2001; 91:1922-5.

35. Inter-American Commission on Human Rights. Case 12.191. Report No. 66/00 María Mamérita Mestanza Chávez. Washington DC: Inter-American Commission on Human Rights; 2000.

36. Miranda J, Baertl U. Derechos humanos y salud: la experiencia de EDHUCASalud. In: Memorias del Primer Taller Latinoamericano Permanente "Derechos Humanos y Salud: Encontrando los Lazos". Lima: Asociación Civil para la Educación en Derechos Humanos con Aplicación en Salud; 2001. p. 99-101.

37. Miranda JJ, Yamin AE. A past, present, and future challenge for the health and human rights movement. Human Rights Dialogue 2002; 2:26.

38. Miranda JJ, Yamin AE. Where is the real debate on globalisation? J Epidemiol Community Health 2002; 56:719.

Recibido el 12/Feb/2007

Aprobado el 05/Jun/2007 